\title{
Parametric Study on Efficient Formation Flying For A Blended-Wing UAV
}

\author{
Hyo-Sang Shin \\ SATM \\ Cranfield University \\ Cranfield, United Kingdom \\ Email: h.shin@cranfield.ac.uk
}

\author{
Antonis F Antoniadis \\ SATM \\ Cranfield University \\ Cranfield, United Kingdom \\ Email: a.f.antoniadis@cranfield.ac.uk
}

\author{
Antonios Tsourdos \\ SATM \\ Cranfield University \\ Cranfield, United Kingdom \\ Email: a.tsourdos@cranfield.ac.uk
}

\begin{abstract}
This paper investigates aerodynamic performance improvements of formation flight at transonic speeds for a medium size Unmanned Aerial Vehicle (UAV). The metric for assessing the aerodynamic improvement of formation flight is the computed drag. The total drag for each formation configuration is compared with a single UAV, where a final drag reduction percentage is estimated. The evaluation of the aerodynamic performance is conducted by employing an in-house Computational Fluid Dynamics (CFD) solver, grid generation and post processing tools. For critical understanding of the tendency of the formation efficiency depending on main parameters, broad formation configurations are analysed. The parameterisation includes number of aircraft, proximity and formation shape. Full realisation of the benefit predicted would need to be proven in the real world, but there is sufficient confidence to suggest that it exists: the empirical parametric analysis suggests that formation flight can improves aerodynamic performance and formation configuration greatly influence the degree of improvement.
\end{abstract}

\section{INTRODUCTION}

Formation flight has been a topic of research across various scientific sectors. Biologists have studied the energy savings of large birds flying in flocks usually in v-type formation. However, there is not clear scientific interpretation up to this day. Despite of this fact, it is widely believed that formation flying of aircraft could bring tactical advantages in operating multiple aircraft and importantly could improve the aerodynamic efficiency. To validate the hypothesis on the aerodynamic performance improvements from the formation flying in aircraft operation, aerodynamicists have carried out numerous studies.

The evaluation is generally conducted by measuring and computing the flow physics and forces exerted on aircraft, and by computing the aerodynamic improvements of formation flight. For instance, a comprehensive study has been performed by NASA [1] where two F/A-18 aircraft flying in tight formation reached a $20 \%$ drag reduction and $18 \%$ fuel savings. The difference between tight and extended flight formations lies with the stream-wise proximity, usually an extended formation is greater than 10 wing-spans, a tight formation is much smaller.

Recently, several studies have been published in conferences and journals presenting aerodynamic studies for both tight and extended UAV formation flight. An aerodynamic performance for extended formation is conducted by Ning et al. [2] where $30 \%$ drag reduction is achieved with two aircraft and $40 \%$ with three aircraft. An inviscid CFD simulation was performed by Kless et al. [3], where extended formation flight of two aircraft was studied with drag reduction of $54 \%$ and $35 \%$ for subsonic and transonic flows, respectively. A study conducted by Kaden and Luckner [4] employed a discrete vortex filament method to model wake vortex roll-up for tight formation flight where it was concluded that the inclusion of fluid physics such as viscosity Reynolds number are required for verification and validation of current modelling approaches for formation flight.

Most of these studies, if not all, considered only a small number of aircraft in formation, generally one to three aircraft. Considering the complexity of the analysis and typical number of manned aircraft in formation for military and civilian applications, the investigation based on a small number of aircraft could be justifiable. However, it could be different, when considering formation flight of Unmanned Aerial Vehicles (UAVs). Numerous studies have investigated strategic and tactical advantages of having many number of UAVs in formation flying and researchers even started to examine the possibility of operating a UAV swarm, where the number of UAVs has different orders of magnitude to formation. In order to investigate aerodynamic performance improvements in UAV formation flying, it might be important to consider increased numbers of aircraft and several configurations of formation, which could become critical due to the increased number. Under this background, this paper aims to perform an initial investigation on several configurations of UAV formation and analyse the effect of these configurations on the aerodynamic performance.

Formation flight aerodynamic benefits are mainly attributed to induced-drag reduction, which is present due to the wake vortices generated from the leader aircraft's wing tip. The pressure difference between the upper and lower wing drives the flow to roll-up at the wing tips. The following aircraft tries to find the "sweet spot" making use of the leader's wing-tip vortices utilising the pressure gradient to increase his lift.

From a CFD perspective, the most accurate simulation for capturing wake vortex physics is Direct Numerical Simulations 
(DNS) followed by Large Eddy Simulations (LES) which both are prohibited due to their immense computational cost; Reynolds Average Navier-Stokes (RANS) is a fair trade-off between the accuracy and cost, capturing efficiently the main features of the these flows. To the best of our knowledge, even the most advanced 3D CFD formation flight simulations in published studies, employ Euler equations with some kind of vortex correction models without turbulence modelling; thus neglecting several physical aspects related to turbulence. However, wake vortex evolution, roll-up and decay has been extensively studied with the employment of sophisticated fluid dynamics models and methods. Hybrid methods i.e. Detached Eddy Simulation (DES), Scale Adaptive Simulation (SAS), hybrid RANS-ILES are becoming popular as to combine the robustness and speed of RANS models for near body computations and high-resolution methods for the wake vortex formations in the far field [5], [6].

As the main objectives of the current analysis is the modelling of great number of formation types and configurations with as much as seven aircraft, 3D computations even with the Euler's equation (inviscid) would be a tremendous computational effort. For an initial investigation, two-dimensions would be ideal as large number of test-cases can be readily performed in a feasible period of time by exploring several configurations and parameters. However, it will be essential to incorporate the 3D wake vortices and induced draft in the 2D-based investigation. Therefore, this paper applies the RANS where Reynolds number, viscosity and compressibility effects are accounted for. Note that these effects are not considered even in current 3D formation flight simulations and, as discussed, the RNAS provides a fair trade off between the computational cost and accuracy. 2D simulation based on the RANS will provide a good approximation for a initial test phase.

In the analysis, drag reduction percentage is examined for a total of 7 formation configurations. Despite we perform 2D-based investigation, the predicted drag reductions for two and three aircraft are similar with drag reduction estimates of reported studies. The analysis results strongly suggest that configuration parameters, such as number of UAVs in the formation and its shape, significantly influence the degree of the drag reduction. The two types of formation configurations tested are echelon and v-type configurations: from the analysis, the efficient formation is the v-type, followed by the echelon. Note that, recognising the limitations of the methods used, full realisation of the benefit predicted would need to be proven in the real world. However, the parametric study in this paper provides a certain level of confidence to suggest the aerodynamic benefit of formation and its tendency with respect to different configurations.

The paper starts with an overview of the physics involved in transonic aerodynamic flows; the governing equations and turbulence model are detailed. A brief description of the in-house solver with discretisation and numerical schemes is presented followed by the grid generation work. The flight envelope and simulation matrix are explained including a preliminary study on the stream-wise proximity. The aerodynamic benefits of each configuration are assessed in the results and discussion section. Finally, the main outcomes are summarised followed by future work directions, recommendations including preliminary results from a 3D simulation.

\section{Framework MODELLING}

This section is devoted to the governing equations, numerical methods and grid generation, the in-house solver employed for the current simulation is detailed demonstrating current and future capabilities related to formation flight physics.

\section{A. Governing Equations}

The most accurate mathematical expression of fluid dynamics lies with the 3D compressible Navier-Stokes equations, where physical phenomena encountered in formation flight can be accurately predicted i.e. compressibility, boundary layer separation, transition to turbulence, shock-waves, wake formation, evolution and decay.

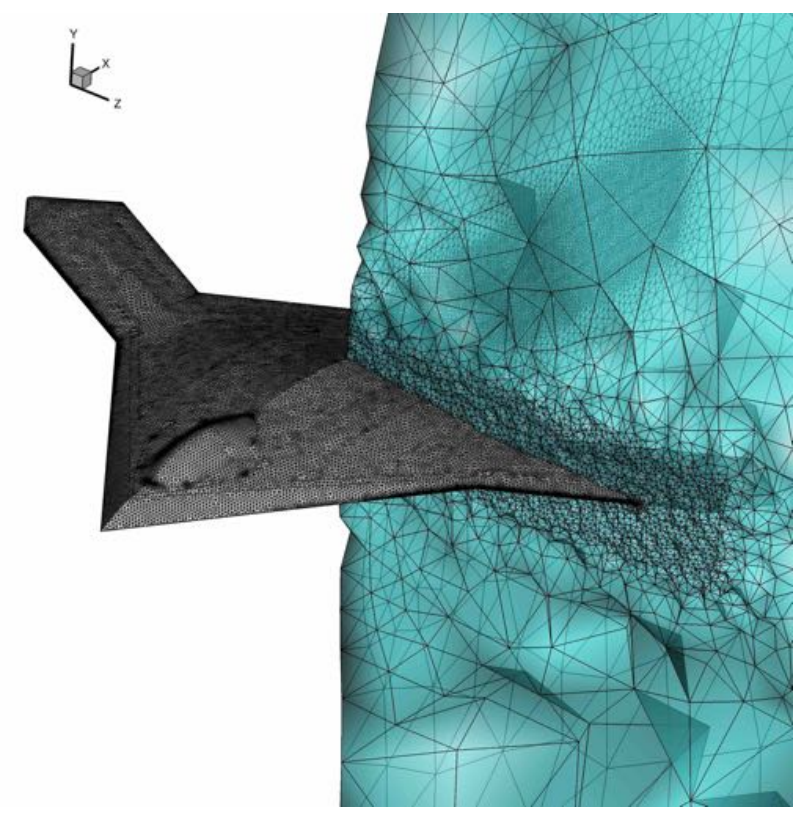

Fig. 1. 3D grid for a typical blended-wing UAV. The grid was generated and composed of approximatly 2 million tetrahedrals, note that the grid is created for half the geometry, here the aircraft surface is mirrored in the $(\mathrm{X}, \mathrm{Y})$ plane for visualisation purposes.

As the main objectives of the current analysis is the modelling of great number of formation types and configurations with as much as ten aircraft, 3D computations even with the Euler's equation (inviscid) would be a tremendous computational effort. To put it in perspective a 3D grid for inviscid simulation (no prism layer) with local refinement in the wake, to be able to capture the wake-vortex, will be composed of approximately 1.5 to 2 millions elements and this will correspond only two half the aircraft with symmetry boundary conditions imposed on the $(\mathrm{X}, \mathrm{Y})$ plane. A 3D grid was generated for a typical blended-wing stealth unmanned combat aerial vehicle shown in Fig. 1. One simulation with this 
grid will take from 1 to 3 days in a modern desktop computer running in six CPUs, depending upon the physics models, numerical algorithms convergence criteria etc. This doesn't include the grid generation procedure which can take up to 2 days for a good quality unstructured 3D grid (1 aircraft).

Two-dimensions would be ideal in the initial investigation as large number of test-cases can be performed in a reasonable period of time by exploring several configurations and parameters. The main drawback of $2 \mathrm{D}$ is the inherited assumption of two-dimensionality where real 3D wake vortices and induced drag effects cannot be modelled. Nevertheless, two-dimensional simulation provides a good approximation for a blind test phase; in addition for the current study the Reynolds Average Navier-Stokes (RANS) are considered where Reynolds number, viscosity and compressibility effects are accounted for; this effect are not considered in current 3D formation flight simulations.

The in-house CFD software solves the RANS equations which are formulated for the finite volume method and discretised on hybrid unstructured elements: triangles and quadrilaterals. The 2D RANS equations are written in integral form as

$$
\frac{\partial}{\partial t} \int_{V_{i}} \mathbf{W}_{\mathbf{i}} d V_{i}+\oint_{\partial V_{i}}\left[\left(\mathbf{F}^{\mathrm{c}}-\mathbf{F}^{\mathbf{v}}\right) \mathbf{n}_{\partial V_{i}}\right] d A_{\partial V_{i}}=0
$$

where $i$ is the index of an element with volume $V_{i}, \mathbf{W}_{\mathbf{i}}$ is the vector of conserved variables, $\mathbf{F}^{\mathrm{c}}$ is the vector of inviscid fluxes and $\mathbf{F}^{\mathrm{v}}$ is the vector of viscous fluxes, the outwardpointing unit normal vector is labelled as $\mathbf{n}=\left(n_{x}, n_{y}\right)$. The equations are dimensionalised according to the free-stream Mach number $M_{\infty}$.

Air is considered as an ideal gas and according to Boussinesq's approximation relating the viscous stress tensor $\tau_{i j}$ to the Reynolds stresses through the eddy viscosity $\mu_{t}$, effectively modelling the momentum transfer by turbulent eddies, written as

$$
\begin{aligned}
\tau_{i j} & =2\left(\mu_{l}+\mu_{t}\right)\left(s_{i j}-\frac{1}{3} \frac{\partial u_{k}}{\partial x_{k}} \delta_{i j}\right), \text { for } i=1,2 j=1,2 \\
s_{i j} & =\frac{1}{2}\left(\frac{\partial u_{i}}{\partial x_{j}}+\frac{\partial u_{j}}{\partial x_{i}}\right)
\end{aligned}
$$

where $k$ is the thermal conductivity, $\delta_{i, j}$ is the Kronecker delta. The eddy viscosity is computed with the Spalart-Allmaras (SA) one-equation turbulence model [7]. The model is widely employed for aerospace application as it is robust across subsonic to supersonic regimes. The model solves one transport quantity the turbulence parameter $\tilde{\nu}$ which is related to eddy viscosity $\mu_{t}$ as

$$
\mu_{t}=\rho \tilde{\nu} f_{v 1} \quad \text { where } \quad f_{v 1}=\frac{\left(\rho \tilde{\nu} / \mu_{l}\right)^{3}}{\left(\rho \tilde{\nu} / \mu_{l}\right)^{3}+C_{v 1}^{3}}, \quad C_{v 1}=7.1
$$

the SA model equation is written as

$$
\begin{aligned}
\frac{d(\rho \tilde{\nu})}{d t}= & C_{b 1} \tilde{S} \rho \tilde{\nu}+\frac{1}{\sigma}\left(\nabla \cdot\left(\mu_{l}+\rho \tilde{\nu}\right) \nabla \tilde{\nu}\right) \\
& +\frac{1}{\sigma} C_{b 2} \rho(\nabla \tilde{\nu})^{2}-C_{w 1} f_{w} \rho\left(\frac{\tilde{\nu} k}{d}\right)^{2}
\end{aligned}
$$

where $C_{b 1}, \tilde{S}, \sigma, C_{b 2}, C_{w 1}, f_{w}, \kappa$ are functions and constants of the model and $d$ is the distance from the nearest wall boundary.

\section{B. Solver Description}

The in-house solver is capable of handling hybrid unstructured grids, where the discretisation scheme evaluate its quantity and gradient according to several numerical schemes. For fast simulations a $1^{\text {st }}$ and $2^{\text {nd }}$-order MUSCL-TVD (Monotone Upstream-centred Schemes for Conservation Laws - Total Variation Diminishing) schemes are used but for higher resolution and accuracy the high-order WENO (Weighted Essentially Non-Oscillatory) schemes can be engaged. Several Riemann solvers are available (HLLC, Roe and Rusanov) where the viscous gradients can be evaluated with either the Green-Gauss or the least-square method. The solver has been extensively validated over a wide range of cases from low-speed flows (Mach=0.08) to shock wave boundary layer flows $(\mathrm{Mach}=5)$, and in a broad spectrum of Reynolds numbers $(\mathrm{Re}=5 \times 10 \mathrm{e} 1$ to $17 \times 10 \mathrm{e} 6)$. Turbulence can accounted with either an ILES approach, RANS models or hybrid methods i.e. (DES and SAS). In addition to the SA turbulence model the K-omega SST is available. The solver is a versatile and portable software written in Fortran-90. It is parallelised with MPI directives and reaches considerable high scalability particularly for higher order ( $>3$ rd) discretisation schemes. An implicit LU-SGS and block Jacobi time advancing scheme can be used for fast and steady-state simulations or an explicit Runge-Kutta scheme for unsteady simulations. Therefore, the in-house solver perfectly fits the objectives of our study. Detailed description of the methods, numerical algorithms and applications can be found in published journal papers [8], [9], [10], [11].

For the current study a first order spatial discretisation is employed where the gradients of temperature, velocities and turbulence viscosity are approximated with the GreenGauss method. The Rusanov Riemann solver [12] handles the computation of the inviscid intercell fluxes as it is very stable for blunt body aerodynamics at transonic speeds. The implicit LU-SGS time discretisation scheme is employed to advance the time to a steady state [13]. Convergence is monitored with the residual of the mean equations and turbulence quantities as well as with the computed drag. Typical convergence behaviour can be depicted from Fig. 2 where the residual histories for 3 UAVs in V-type formation are shown for the density, energy and drag. The solution is assumed to be converged where the drag is stabilised around forty thousand iterations. 


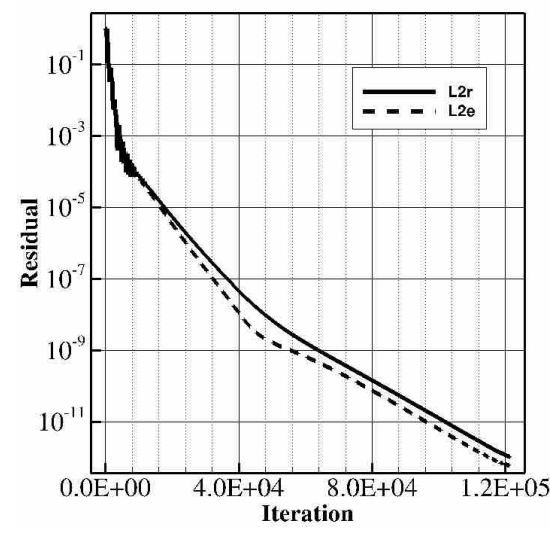

(a) Residual of $\lambda 2$ norm for density and energy

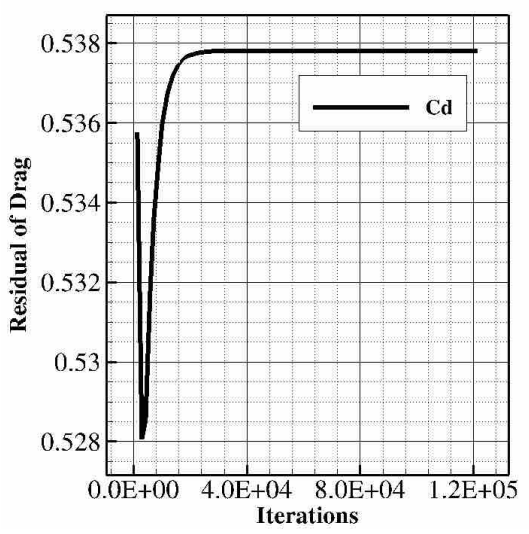

(b) Residual of $C_{d}$

Fig. 2. Residual history for $3 \mathrm{UAVs}$ in V-type formation

\section{Geometry and Grid Generation}

As acknowledged, this study is supported by BAE Systems (Operations). The UAV geometry is based on an approximate design of the BAE-systems blended wing UAV "Corax/Raven" shown in Fig. 3. The wing-span is assumed to be around 10 meters which is used for the estimation of the Reynolds number. Both top and side view 2D simulation with one aircraft are performed, however the side view approach prove to be misleading as the lift remained very small as the simulated 2D plane was set on half way of the wing-span. In addition the side view would limit the number of possible combinations of formation configuration, therefore the top view was selected to perform the CFD analysis. Fig. 4 illustrates the grid for one aircraft with focus both on the body and far-field. Figures 5 and 6 show the grids generated for the echelon and v-type formation configurations, respectively.

Once the geometry (points and curves) are extracted from the figures, they are imported in a grid generation software. Hybrid unstructured grids provide exceptional easy for generation and manipulation of the grid which is ideal for the current analysis. Quadrilateral elements are extruded from the surface and triangular elements are generated for the far-field.

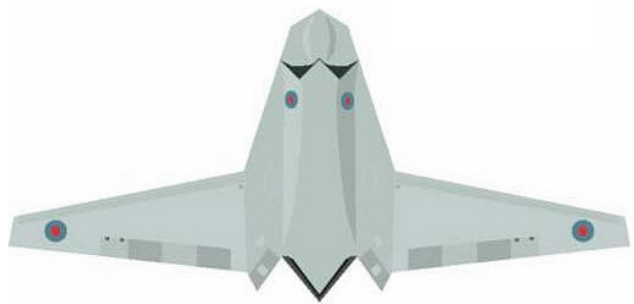

(a) Top view

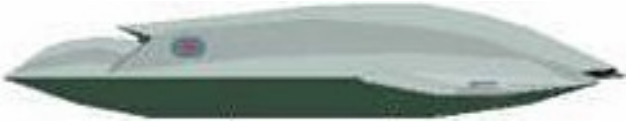

(b) Side view

Fig. 3. Geometry and configuration of the BAE-systems Corax/Raven UCAV (http://www.unicraft.biz/on/corax/corax.htm).

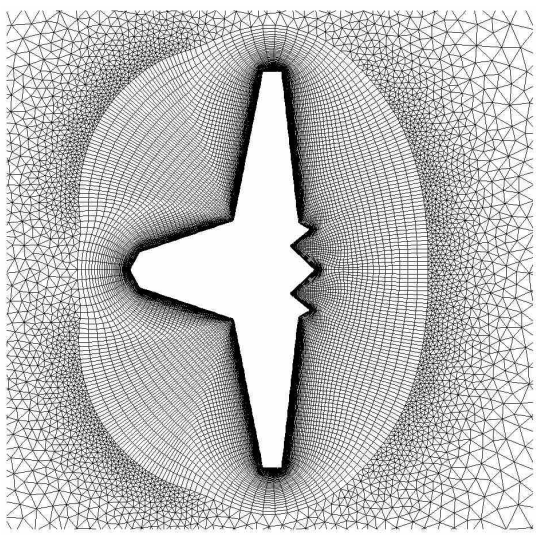

(a) Grid focused on the UCAV

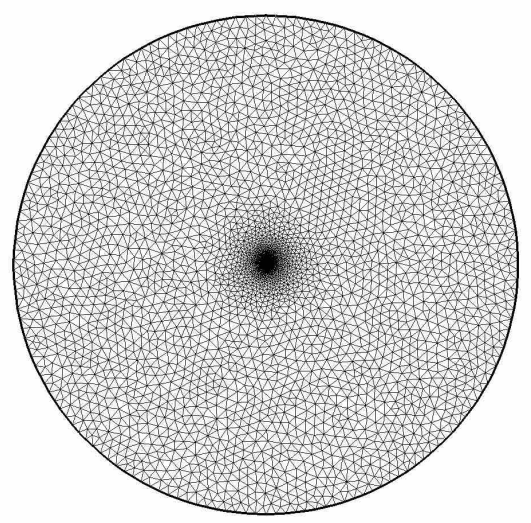

(b) Far-field Grid

Fig. 4. Hybrid unstructured 2D grid for one UAV configuration composed of 29, 962 elements. 


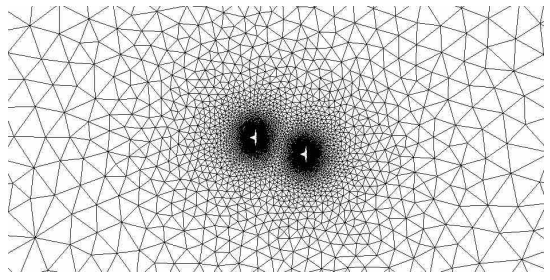

(a) Echelon 2

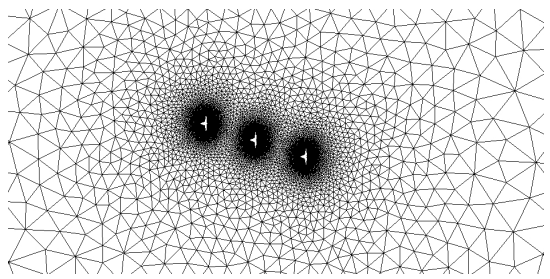

(b) Echelon 3

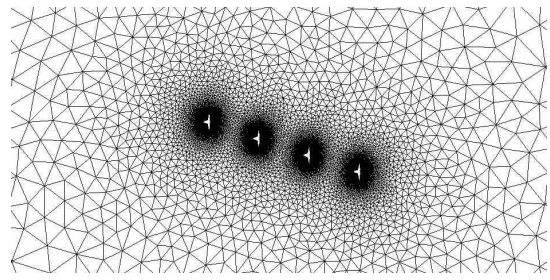

(c) Echelon 4

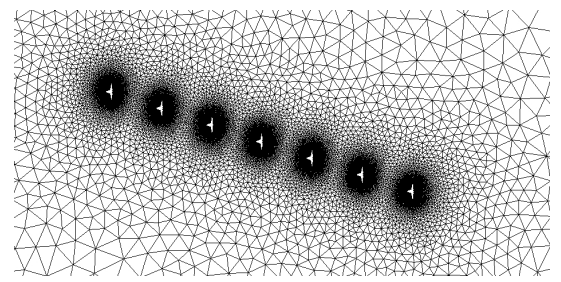

(d) Echelon 7

Fig. 5. Hybrid grids for the echelon formation.

\section{Simulation Set-Up}

This section entails the free-stream conditions, simulation framework, and complete test-case matrix. An initial study is conducted to determine the stream-wise optimal proximity simulating two and three aircraft, once the optimal distance was found it was used for the rest of the test-cases. The aerodynamic efficiency improvement metric is the drag and the results are assessed based on the percentage of drag reduction.

\section{A. Free-Stream Conditions}

A free-stream Mach number of $M_{\infty}=0.7$ is set as freestream condition corresponding to a transonic regime with a corresponding Reynolds number of $R e \approx 75 \times 10^{6}$ based on half of the wing-span. Far-field boundary conditions are imposed on the outer boundaries where flow quantities are computed based on the characteristic speed (local speed of sound).

\section{B. Proximity Analysis}

As two dimensions are considered proximity between aircraft is accounted for displacement in the $\mathrm{x}$ and $\mathrm{y}$ axis. The

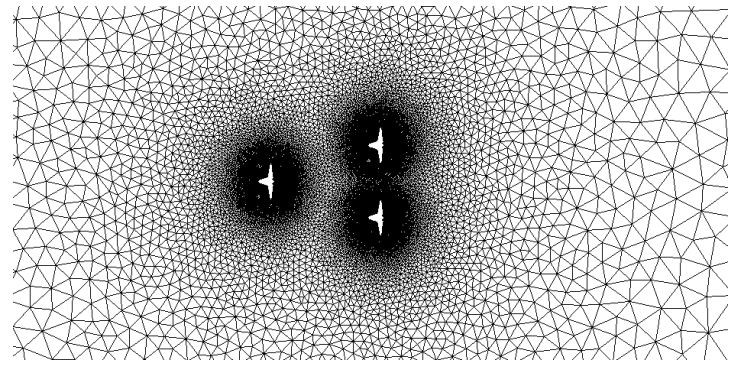

(a) V-type 3

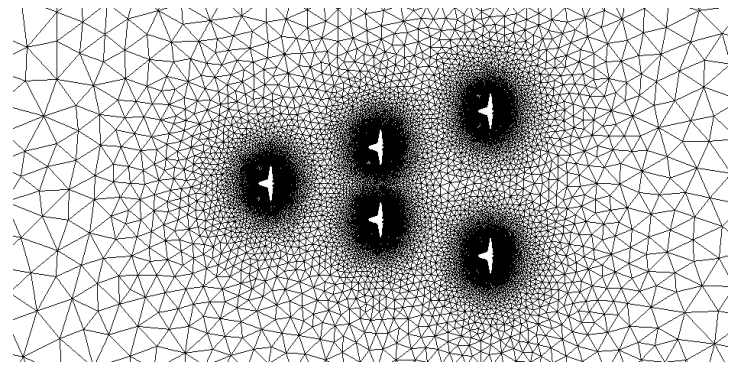

(b) V-type 5

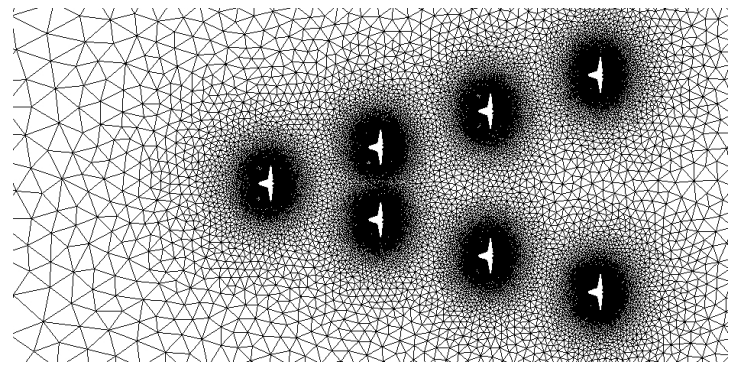

(c) V-type 7

Fig. 6. Hybrid grids for V-type formation.

y proximity is set at wing tip-to-tip distance of zero. Tipto-tip distance can be negative as well, where the wing-tip of the following aircraft is positioned further inside on the leader's aircraft's wake. It has been reported in the literature that a small tip-to-tip distance will produces the greatest drag reduction for both extended and tight formations [4], [14].

For x-proximity a sensitivity analysis is performed with two aircraft in order to establish the stream-wise distance of aircraft. Four distances are assessed: 1,2,3 and 4 wingspans, Fig. 7 shows the four configurations and and the vortical structure downstream with streamlines. 1 wing-spans will produce the least drag reduction followed by the 2 with $13.78 \%$ and $23.32 \%$ respectively. Increasing the distance to 3 wing-spans further increases the drag reduction by $26.45 \%$ and with 4 wing-spans to $27.21 \%$. The greatest difference is estimated to be between case 1 and 2, where further increasing the $\mathrm{x}$-distance seems to have an asymptotic trend. In addition, three aircraft simulation on v-type formation are performed with a uniform streamwise distance of 1 and 3 wing-spans. The drag reduction with the greatest distance (3 wing-spans) showed an improvement of drag reduction of 1.7 times the improvement with the 1 wing-span proximity. Therefore, a 


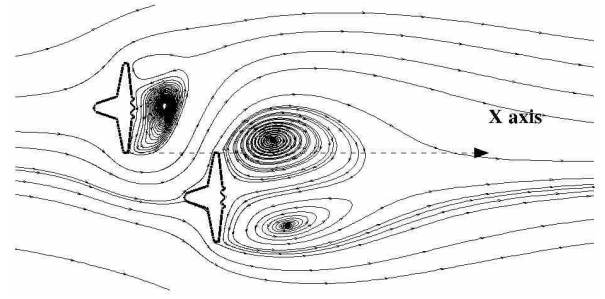

(a) 1 wing-span

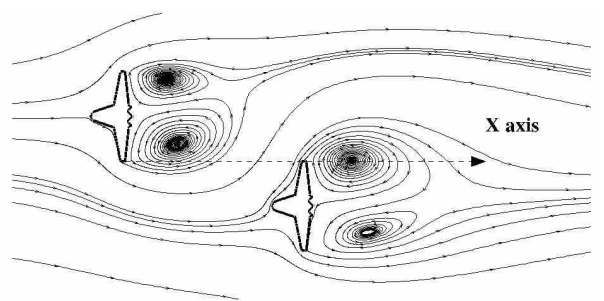

(b) 2 wing-span

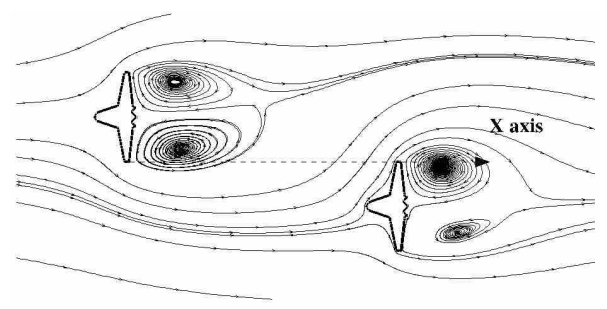

(c) 3 wing-span

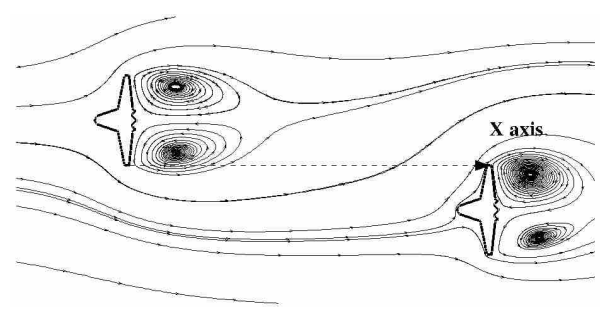

(d) 4 wing-span

Fig. 7. Proximity analysis for $\mathrm{x}$-axis performed with four different wingspans, plotted streamlines for visualising the flow field patterns.

uniform three wing-spans streamwise proximity is selected to carry out the complete test-case matrix which is also in-line with tight formation requirement.

\section{Simulation Matrix}

A total of 8 test-cases are simulated, where for consistency a uniform streamwise and tip-to-tip distance is maintained for all aircraft and formation shapes in detail: 5 echelon and 3 v-type configurations are simulated. The detailed parameters are summarised in Table I, containing information for each test-case on the formation shape, number of aircraft, number of grid elements and drag reduction percentages.

\section{RESUlts AND Discussion}

This section presents the results from the CFD simulations, quantitatively in terms of drag reduction percentages as well
TABLE I

SIMULATION MATRIX

\begin{tabular}{ccccc} 
Test-Case & Formation Shape & aircraft & $\begin{array}{c}\text { Grid } \\
\text { Elements }\end{array}$ & $\begin{array}{c}\text { Drag } \\
\text { Reduction }(\%)\end{array}$ \\
\hline 1 & - & 1 & 29,962 & - \\
2 & Echelon & 2 & 54,146 & $26.45 \%$ \\
3 & Echelon & 3 & 78,538 & $30.96 \%$ \\
4 & Echelon & 4 & 102,864 & $34.21 \%$ \\
5 & Echelon & 7 & 175,530 & $44.08 \%$ \\
6 & V-type & 3 & 96,350 & $34.84 \%$ \\
7 & V-type & 5 & 125,906 & $42.24 \%$ \\
8 & V-type & 7 & 182,544 & $47.84 \%$ \\
\hline
\end{tabular}

as qualitatively with the use contours plots of Mach number and eddy viscosity.

The benefits of formation flight depended upon the formation shape and number of aircraft is assessed with the total drag for each test-case divided by the number of aircraft in formation and compared with the drag of one aircraft. As expected, increasing the number of aircraft reduces the drag for all formation shapes. The results show that the most efficient formation among the two is the v-types reaching up to about $48 \%$ drag reduction with 7 aircraft whereas echelon $44 \%$ for the same aircraft number.

The echelon formation is the less efficient, which can be attributed to its sparse configuration. The v-type formation commonly encountered in nature (geese and large birds) is more efficient than the echelon.

Even if this analysis is $2 \mathrm{D}$ at specific flight conditions similar drag reductions are reported from other studies, NASA report [1] presents a $20 \%$ with two aircraft at tight formation where the current analysis estimated a $26 \%$. Ning et al. [2] predicted $30 \%$ for two aircraft and $40 \%$ with three, similar reduction is observed with this study, where three aircraft (V-type) have a $35 \%$ with a reduction difference of $8.5 \%$ compared with $10 \%$ going from two to three aircraft.

The reduction of drag can be correlated also qualitatively with the amount of eddy viscosity computed by the turbulence model. Higher eddy viscosity values in the wake, correspond usually to a more turbulent flow which in terms can have an effect on the computed drag.

\section{CONCLUSIONS AND FUTURE WORK}

This paper comprises 2D CFD computations for UAV flight formation at transonic speeds. Echelon and V-type formation shape are assessed by also varying the number of aircraft (2 7). To establish stream-wise proximity distance, an analysis is conducted where three wing-span distance was selected for uniform stream-wise spacing. A total of 7 configurations are simulated where drag reduction percentage have been estimated. From the study it was found that the more efficient formation is the v-type formation, followed by the the echelon. The predicted drag reductions for two and three aircraft are similar with drag reduction estimates of reported studies.

A 3D inviscid simulation was performed employing the grid shown in Fig. 1, at a Mach number $M_{\infty}=0.7$ and an angle of attack of 1.5. Initial results are shown in Figures 
8,9 and 10 where contours of the pressure distribution are plotted on the aircraft surface and $w$ velocity is used as a mean of identify the wing tip vortex location. More advanced method of identify wing-tip vortices can be employ i.e. qcriterion, $\lambda 2$ and helicity. Furthermore a prismatic 3D grid will enable boundary layer prediction by capturing separation, and transition phenomena.

Considering the limitations of the methods used, we might need to prove the benefit of the formation flight and its degree with respect to formation configurations through full realisation. However, this initial study provides a certain level of confidence on the aerodynamic benefit of the formation flying and its dependency to the configuration parameters.

Future routes can have several interesting directions which can even run in parallel with each other.

- Perform 2D simulations exploring more formation configuration, combinations and increasing the number of aircraft;

- 2D simulations with non-uniform stream-wise proximity;

- Based on the two previous studies, construct optimisation algorithm to determine the optimal stream-wise proximity distance for a given number of aircraft;

- 2D simulation (top-view) of 3D geometry;

- 3D RANS simulation of single aircraft;

- 3D RANS simulation of two and more aircraft and validate $2 \mathrm{D}$ results.

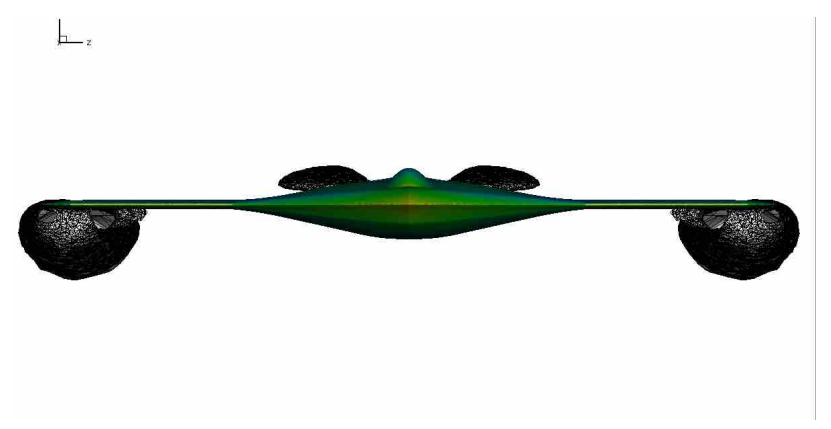

(a) Front view

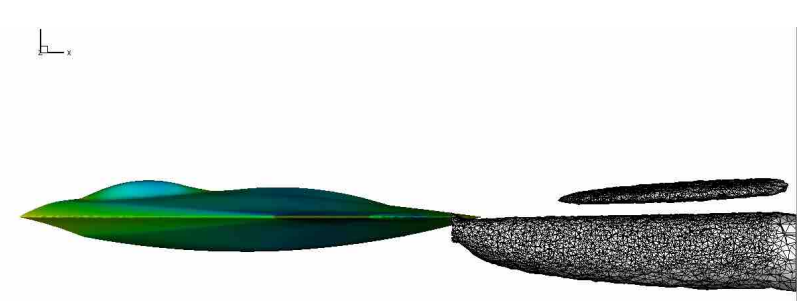

(b) Side view

Fig. 8. Surface pressure distribution and isosurface of w velocity illustrating also the volume grid spacing.

\section{ACKNOWLEDGEMENT}

This work was fully supported by BAE Systems (Operation), who have reviewed and agreed the contents of this paper.

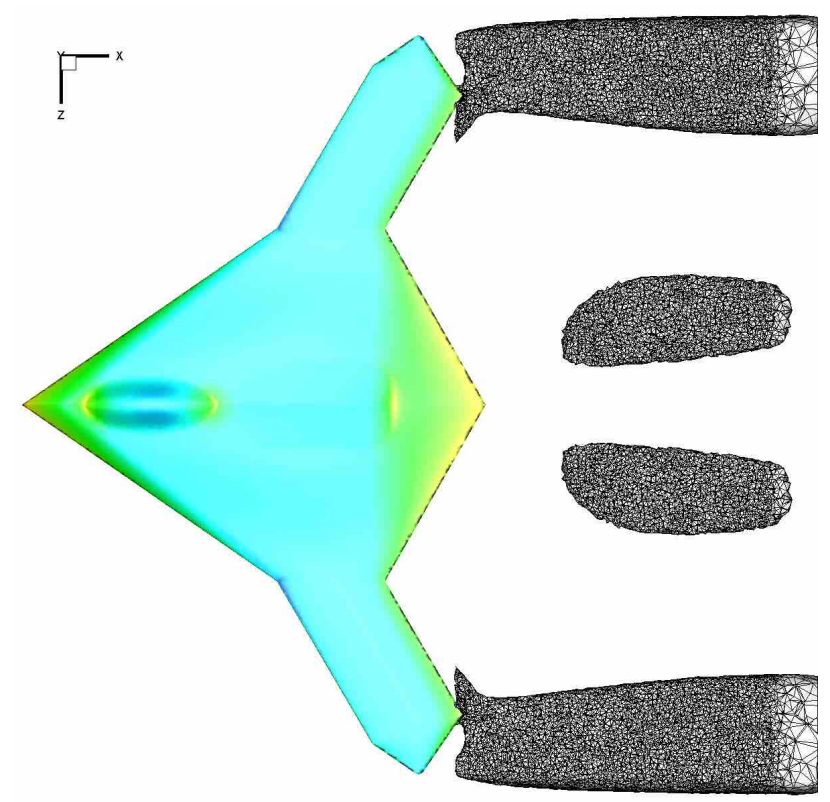

Fig. 9. Surface pressure distribution and isosurface of w velocity illustrating also the volume grid spacing - top view.
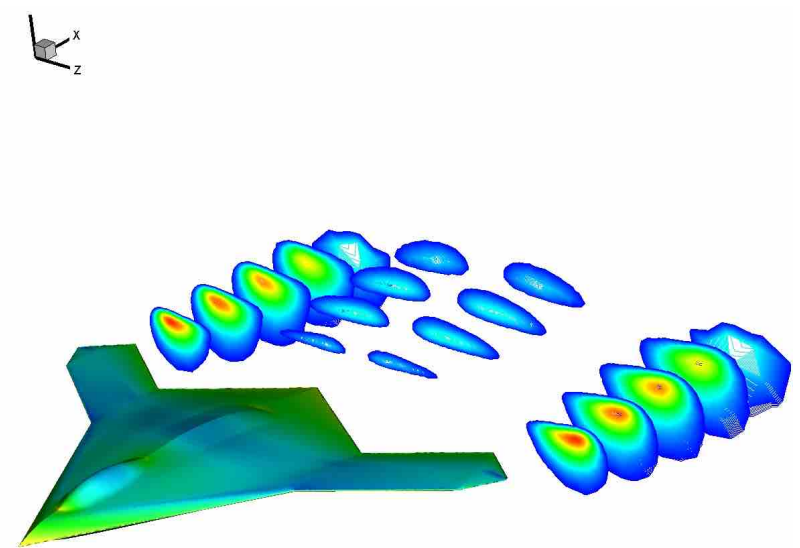

Fig. 10. Surface pressure distribution and contour lines of w velocity.

\section{REFERENCES}

[1] M. Vachon, K. Ray, R.J. Walsh, and K. Ennix, "F/A-18 performance benefits measured during the autonomous formation flight project," National Aeronautics and Space Administration, Tech. Rep. NASA/TM2003-210734, 2003.

[2] S. A. Ning, T. C. Flanzer, and I. M. Kroo, "Aerodynamic performance of extended formation flight," Journal of Aircraft, vol. 48, no. 3, pp. 855-865, 2011.

[3] J. E. Kless, M. J. Aftosmis, S. A. Ning, and M. Nemec, "Inviscid analysis of extended-formation flight," AIAA Journal, vol. 51, no. 7, 2013.

[4] A. Kaden and R. Luckner, "Modeling wake vortex roll-up and vortex- 
induced forces and moments for tight formation flight," in AIAA Modeling and Simulation Technologies (MST) Conference, AIAA, Ed., 2013.

[5] T. Gerz and T. Ehret, "Wingtip vortices and exhaust jets during the jet regime of aircraft wakes," Aerospace Science and Technology, vol. 1, no. 7, pp. 463-474, 1997.

[6] T. Misaka, F. Holzapfel, and T. Gerz, "Wake evolution of high-lift configuration from roll-up to vortex decay," in 51st AIAA Aerospace Sciences Meeting including the New Horizons Forum and Aerospace Exposition 2013, AIAA, Ed., 2013.

[7] P. R. Spalart and S. R. Allmaras, "One-equation turbulence model for aerodynamic flows," Recherche aerospatiale, no. 1, pp. 5-21, 1994.

[8] A. Antoniadis, P. Tsoutsanis, and Drikakis.D., "High-order schemes on mixed-element unstructured grids for aerodynamic flows," in 42nd AIAA Fluid Dynamics Conference and Exhibit, AIAA, Ed., no. AIAA 20122833, New Orleans, Louisiana, June 2007.

[9] P. Tsoutsanis, V. A. Titarev, and D. Drikakis, "WENO schemes on arbitrary mixed-element unstructured meshes in three space dimensions," Journal of Computational Physics, vol. 230, no. 4, pp. 1585-1601, 2011.

[10] V. A. Titarev, P. Tsoutsanis, and D. Drikakis, "WENO schemes for mixed-element unstructured meshes," Communications in Computational Physics, vol. 8, no. 3, pp. 585-609, 2010.

[11] P. Tsoutsanis, A. F. Antoniadis, and D. Drikakis, "WENO schemes on arbitrary unstructured meshes for laminar, transitional and turbulent flows," Journal of Computational Physics, vol. 256, pp. 254-276, 2014.

[12] V. Rusanov, "Calculation of interaction of non-steady shock waves with obstacles," J. Comput. Math. Phys., vol. 1, no. USSR, pp. 267-279, 1961.

[13] H. Luo, J. D. Baum, and R. Löhner, "A fast, matrix-free implicit method for compressible flows on unstructured grids," Journal of Computational Physics, vol. 146, no. 2, pp. 664-690, 1998.

[14] J. Pahle, D. Berger, M. Venti, C. Duggan, J. Faber, and K. Cardinal, "An initial flight investigation of formation flight for drag reduction on the c-17 aircraft," in AIAA Atmospheric Flight Mechanics Conference 2012, AIAA, Ed., 2012. 
2017-12-31

\section{Parametric study on efficient formation flying for a blended-wing UAV}

Shin, Hyo-Sang

IEEE

Hyo-Sang Shin, Antonis F. Antoniadis and Antonios Tsourdos. Parametric study on efficient formation flying for a blended-wing UAV. Proceedings of the 2017 International Conference on Unmanned Aircraft Systems (ICUAS), 13-16 June 2017, Miami, USA. pp. 1657-1664 https://doi.org/10.1109/ICUAS.2017.7991453

Downloaded from Cranfield Library Services E-Repository 